\title{
Numerical and experimental study of a nearby solid boundary and partial submergence effects on hydrofoil added mass
}

\author{
O. De La Torre ${ }^{a, *}$, X. Escaler ${ }^{a}$, E. Egusquiza ${ }^{a}$, M. Farhat ${ }^{b}$ \\ ${ }^{a}$ Center for Industrial Diagnostics, Universitat Politècnica de Catalunya, Av. Diagonal 647, 08028 Barcelona, Spain \\ ${ }^{\mathrm{b}}$ Laboratory for Hydraulic Machines, École Polytechnique Fédérale de Lausanne (EPFL), Lausanne, Switzerland
}

\section{A R T I C L E I N F O}

\section{Article history:}

Received 7 August 2013

Received in revised form 29 November 2013

Accepted 5 December 2013

Available online 11 December 2013

\section{Keywords:}

Added mass

Natural frequency

Boundary condition

FEM

\begin{abstract}
A B S T R A C T
The influence of the boundary conditions on the added mass of a NACA0009 cantilever hydrofoil has been numerically and experimentally investigated. The study has been focused on the effects of a lateral solid wall close to the hydrofoil tip side and of a partially wetted vertical hydrofoil at different levels with the free surface parallel to the span. A detailed fluid-structure model has been built for both cases and a modal analysis has been carried out. The numerical three first natural frequencies have been used to calculate the added mass coefficients. These coefficients have been compared with the results of the analogous experiments carried out by means of an onboard excitation and response system based on piezoelectric patches. The obtained results are in reasonable good agreement with experimental data. The proximity of a lateral solid wall provokes an increase of the added mass experienced by the hydrofoil which can be approximated by a 2 nd order polynomial function for both bending and torsion modes. On the other hand, the increase of the added mass with the level of partial submergence is found to be dependent on the relative displacements of the hydrofoil submerged part and hence on the mode shape.
\end{abstract} (c) 2013 Elsevier Ltd. All rights reserved.

\section{Introduction}

\subsection{Background}

Boundary conditions have a paramount role in the calculation of the added mass of any submerged structure. Their effect on the added mass, and hence on the natural frequencies, differs depending on the considered boundary condition and on multiple others variables.

The study of the added mass of a submerged structure has focused great attention for a long time. Great theoretical, experimental and computational efforts have been devoted to this topic $[13,2,7]$ and from the very beginning the authors realized about the significance of the problem's boundary conditions. Even when Blevins [1] published a collection of formulas referred to natural frequencies and mode shapes, several of them referenced to the effect of lateral walls. Also Brennen [2] mentioned the effect of a nearly solid boundary and a free surface when reviewing the added mass.

On the other hand, from a computational point of view, the accurate calculation of the added mass means great savings both in terms of time and funding in many industrial applications. The reliability of many submerged bodies is based on the accurate prediction of their natural frequencies under actual conditions.

\footnotetext{
* Corresponding author. Tel.: +34 9340159 45; fax: +34 934015812 .

E-mail address: oscar.de.la.torre@mf.upc.edu (O. De La Torre).
}

For instance, Liang et al. [12] simulated the effects of the added mass on the natural frequencies of a whole Francis turbine runner previously experimentally studied by Rodriguez et al. [16]. Also Huang et al. [6] studied numerically and experimentally the added mass of a submerged trash-rack with satisfactory results. In fact, this topic has been of great interest for a long time when related to basic-shape structures. In this sense, Kwak [8] and Liang et al. [10] studied the effect of the surrounding fluid on the natural frequencies of circular plates calculating the added virtual mass incremental (AVMI) factors. Also Liang et al. [11] presented a procedure to determine the natural frequencies and mode shapes of submerged cantilever plates.

The study presented in this paper derives from a wider experimental investigation partially published in [3]. The experimental results shown here were obtained when carrying out the experimental campaign to study the added mass effects for different flow configurations with and without leading edge cavitation and supercavitation. During this investigation, the authors realized about the importance of the selected boundary conditions on the obtained added mass results and decided to characterize them more in detail from both the numerical and the experimental points of view.

\subsection{Added mass effects}

When submerged in a fluid, the dynamic response of a solid body is altered by the effect of the added mass of the fluid, $A_{\text {fluid }}$. 


\section{Nomenclature}

EPFL Ecole Polytechnique Fédérale de Lausanne

FEM finite element method

FSI fluid structure interaction

LE leading edge

$\begin{array}{ll}\text { PZT } & \text { lead zirconate titanate } \\ \text { TE } & \text { trailing edge } \\ C_{M} & \text { added mass coefficient } \\ f & \text { natural frequency }\end{array}$

Consequently, the ratio between the natural frequency of a given mode of vibration in water, $f_{\text {fluid }_{i}}$, and in air, $f_{\text {vacuum }_{i}}$, is approximately given by:

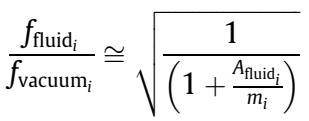

where $i$ subscript denotes each particular mode shape and $m$ is the corresponding modal mass. As a result, the natural frequencies of vibration in water are lowered with respect to those in air (considered as a vacuum). If the added mass matrix is diagonal, the system mode shapes in water are equivalent to those of the structure vibrating in air.

From Eq. (1), an added mass coefficient, $C_{M}$, can be defined with Eq. (2). This coefficient will be of special interest throughout the present work because it is used to quantify the added mass effects.

$C_{M_{i}}=\left(\frac{f_{\text {vacuum }_{i}}}{f_{\text {fluid }_{i}}}\right)^{2}-1$

\subsection{Boundary conditions under study}

\subsubsection{Wall effect}

This first boundary condition is presented when a rigid wall is located nearby the vibrating structure. In this situation, the added mass matrix can be simplified with geometric symmetries if, and only if, the symmetries are applied to both the structure under study and the solid wall.

Using potential flow theory, the system of a solid wall near a body was studied by Lamb [9] and Milne-Thomson [14], who modified the kinetic energy of an unbounded sphere to take into account the energy increase produced by the wall effect. Time after, it was observed that the presence of a solid wall near a vibrating structure could cause a substantial increase in the added mass $[18,20]$. In the region between the structure and the boundary, the fluid experiences an acceleration increase, which enhances the inertial forces exerted on the wetted surfaces. As the structure moves away from the boundary, the added mass rapidly decreases asymptotically to the value of an isolated body.

When referring to numerical simulations, Motley et al. [15] studied the effect of several boundary conditions - solid wall, free surface and partial submergence- on the natural frequencies of different composite plates and its dependency with the fiber angle. In this work, the authors concluded that solid boundaries had limited effects for fully-submerged plates near a wall. Also Rodriguez et al. [17] studied the effect of a close solid boundary on the added mass of a rectangular plate. Anyhow, the authors of this paper were basically focused on validating a structural-acoustic FSI numerical method as an alternative to experimental tests.

\subsubsection{Partial submergence}

The second boundary condition corresponds to the effect of having the body partially submerged with a free surface in contact with it. In this sense, Lindholm's et al. [13] work has been taken as a reference to design the current tests. This specific case is of paramount interest in the naval industry where this condition is present in multiple systems and components like the propellers in different situations or even in the study of the whole ship.

The resonance frequencies of vertical and horizontal cantilever plates partially or totally submerged in water were also studied by Fu and Price [5] but limited experimental comparison was given. More recently, Ergin and Ugurlu [4] studied the effect of partial submergence on the natural frequencies and mode shapes of different cantilever plates and successfully compared the numerical results with experimental data.

\subsection{Objectives}

In this paper, the effects of a nearby solid wall and of partial submergence on the natural frequencies of a cantilever NACA0009 hydrofoil have been studied separately. In particular, numerical simulations of both effects have been carried out and the results have been compared with the experimental ones. As a result, the validity of a commercial multiphysics software (Ansys ${ }^{\circledR}$ ) as a reliable tool in the resolution of this kind of cases has been checked.

The study mainly focuses on the first three natural frequencies of the tested hydrofoil which in the case of the tested hydrofoil correspond to the first and second bending modes $\left(f_{1}\right.$ and $f_{3}$ respectively) and first torsion mode $\left(f_{2}\right)$ as observed in Fig. 1 .

\section{Experimental methodology}

The experimental methodology is analogous to the one described in De La Torre et al. [3]. In this previous work, the authors carried out an extensive investigation of the added mass under different flow conditions in the test section of the EPFL High Speed Cavitation Tunnel. The test section, which is shown in the Fig. 7, has a rectangular shape and $150 \times 150 \times 750 \mathrm{~mm}$ as inner dimensions. A 7075 aluminum type hydrofoil with a $150 \mathrm{~mm}$ span and $100 \mathrm{~mm}$ chord was used. This particular material has a density of $2800 \mathrm{~kg} / \mathrm{m}^{3}$ and a Young's modulus of $72 \mathrm{GPa}$. The trailing edge was cut at $45^{\circ}$. In order to experimentally obtain the hydrofoil's natural frequencies an innovative PZT excitation and measuring system was embedded on its surface. Two PZT patches were mounted in the particular locations shown in Fig. 2 that permitted to excite the hydrofoil bending and torsion modes. The hydrofoil installation within the tunnel test section consisted in clamping one side to a lateral wall, obtaining a cantilever structure configuration as observed in Fig. 3.

In order to excite the structure, a signal generator fed the PZT patch closer to the leading edge through an amplifier. By exciting a wide frequency band with a 120-s sinusoidal chirp signal and measuring at the same time the response with the trailing edge patch, it was expected to identify the response amplification under resonance conditions. The chirp signals covered a frequency band of $240 \mathrm{~Hz}$ with a constant frequency increase factor of $2 \mathrm{~Hz} / \mathrm{s}$. The frequency range excited in each chirp could differ depending on the mode sought or the conditions in the test section. The acquired data was conditioned and recorded by a 16 byte A/D system with eight simultaneous input channels and a selectable sampling frequency with a maximum of 250000 samples per segment. 

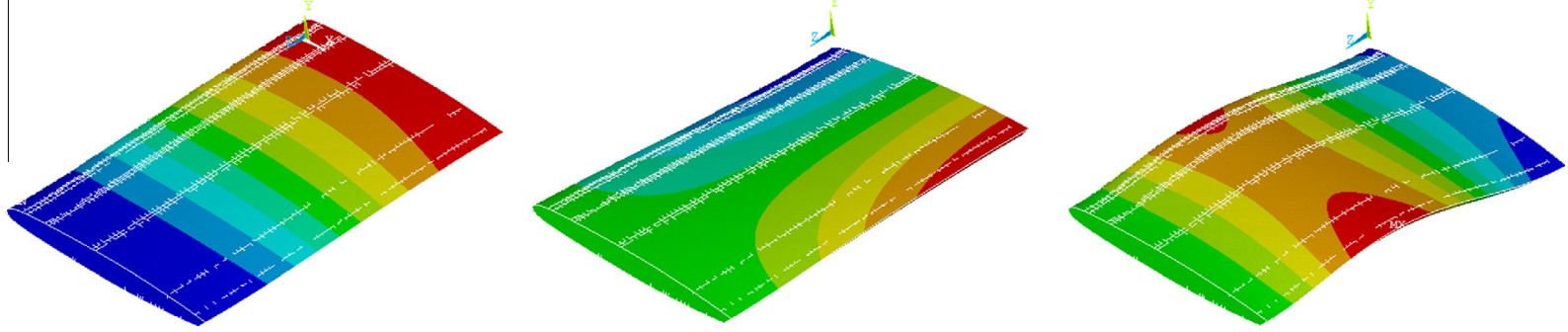

Fig. 1. Mode shapes under study: first bending (left), first torsion (middle) and second bending (right).

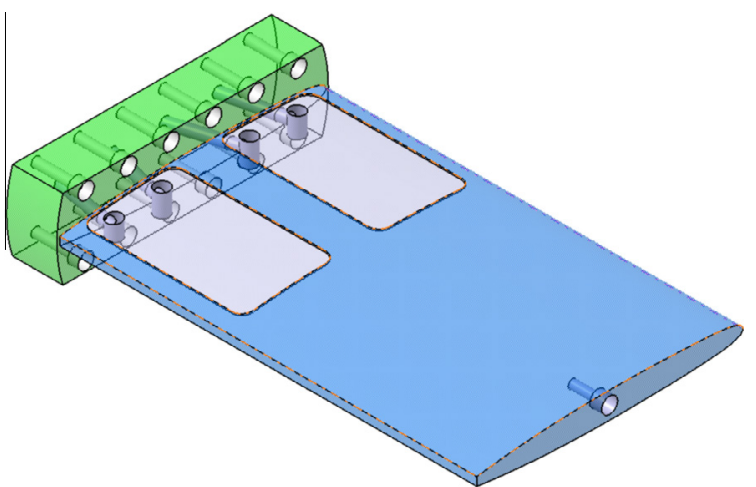

Fig. 2. Sketch of the PZT excitation system installed on a NACA0009 hydrofoil.

\subsection{Lateral gap analysis}

The lateral gap was increased from a minimum value of $0.12 \mathrm{~mm}$ to a maximum of $2.12 \mathrm{~mm}$ by means of different metallic plates of calibrated thickness, which were placed between the hydrofoil subjection system and the tunnel wall. For each gap distance, modal analyses were performed following the same procedure explained in De La Torre et al. [3].

\subsection{Partially submerged hydrofoil}

The profile was located perpendicular to the free surface of the water, and the tunnel was filled in stages. A modal analysis was performed from $0 \%$ to $100 \%$ submergence for each $10 \%$ submergence variation. Additionally, because the profile is non-symmetric, the two different orientations were taken into account by analyzing the body with the leading edge or the trailing edge submerged, as observed in Fig. 4.

\section{Numerical methodology}

The numerical methodology started with the main model setup to assure the validity of the results, and two different cases were subsequently simulated. First, the effects of the distance of the test section lateral wall to the lateral hydrofoil tip section, indicated as the gap on the right of Fig. 3, were studied. Second, the effects of the partial submergence conditions with two different hydrofoil orientations as indicated in Fig. 4 were also studied.

\subsection{FSI equations}

Under the acoustics field, the structural dynamics equation must be considered together with the Navier-Stokes equations. The fluid domain requires the following assumptions to simplify the analysis:

1. The fluid is compressible (due to pressure variations).

2. The fluid is inviscid.

3. There is no mean fluid flow.

4. The mean density and pressure are uniform throughout the fluid.

In this way, we obtain the acoustic wave in the following equation:
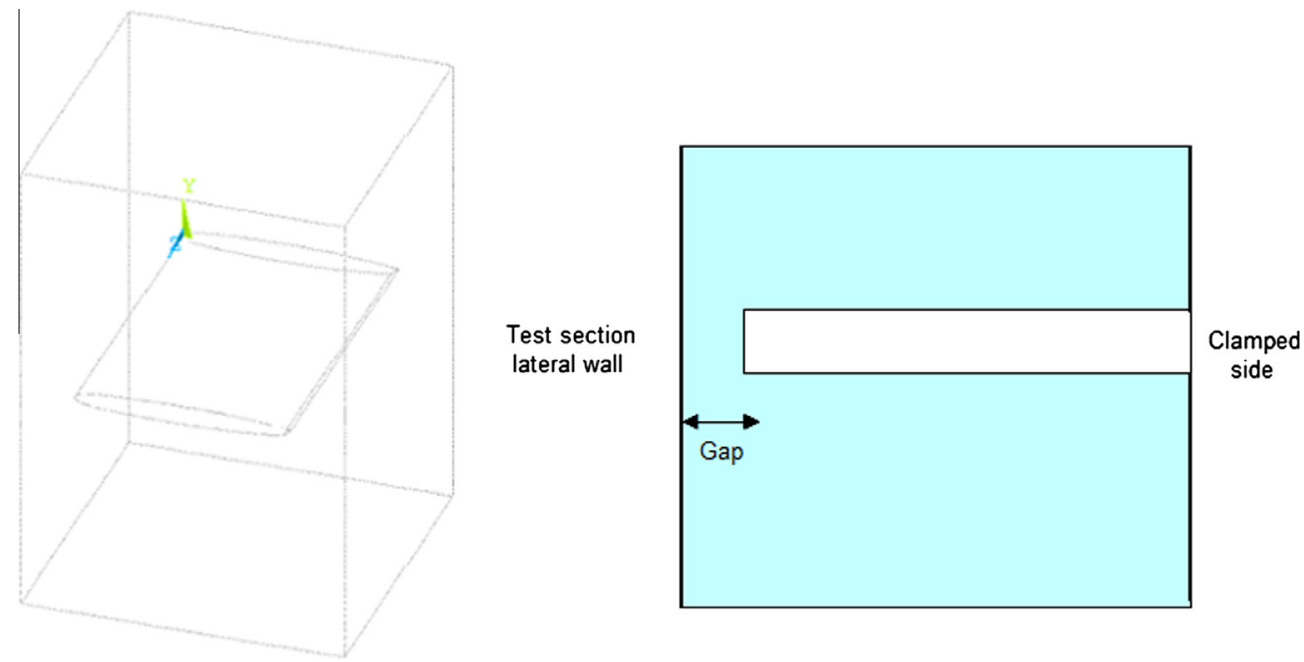

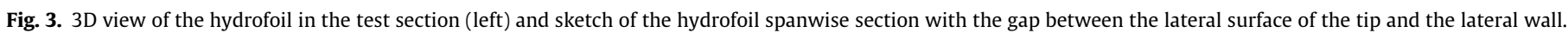




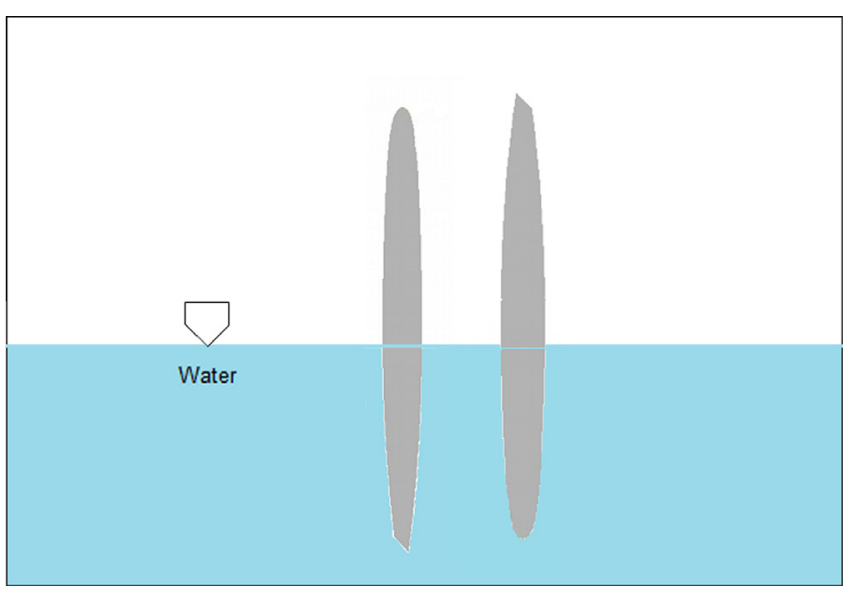

Fig. 4. Sketches of the hydrofoil chordwise section with trailing edge (left) and leading edge (right) orientations under partial submergence conditions.

$\frac{1}{c^{2}} \frac{\partial^{2} P}{\partial t^{2}}-\nabla^{2} P=0$

where $c$ is the speed of sound in the fluid, $P$ is the pressure and $t$ is the time. Eq. (3) and the structural dynamic Eq. (4) constitute the FSI system solved by FEM.

$y=\frac{F_{o}}{\sqrt{\left(k-m \omega^{2}\right)^{2}+(c \omega)^{2}}} \cos (\omega t+\varphi)+e^{-\frac{c}{2 m} t}\left(C_{1} \cos \left(\omega_{d} t\right)\right.$

Because the fluid is modeled as inviscid, a dissipation term is added to account for the dissipation of energy due to the damping effect in the fluid boundary. Next, both Eqs. (3) and (4) are discretized, leading to a FSI system such as the one represented by:

$$
\left[\begin{array}{ll}
M_{e} & 0 \\
M^{f s} & M_{e}^{p}
\end{array}\right]\left[\begin{array}{l}
\ddot{u}_{e} \\
\ddot{P}_{e}
\end{array}\right]+\left[\begin{array}{ll}
C_{e} & 0 \\
0 & C_{e}^{p}
\end{array}\right]\left[\begin{array}{l}
\dot{u}_{e} \\
\dot{P}_{e}
\end{array}\right]+\left[\begin{array}{ll}
K_{e} & 0 \\
0 & K_{e}^{p}
\end{array}\right]\left[\begin{array}{l}
u_{e} \\
P_{e}
\end{array}\right]=\left[\begin{array}{l}
F_{e} \\
0
\end{array}\right]
$$

where $M_{e}, C_{e}$ and $K_{e}$ are the mass, damping and stiffness matrices, respectively, and $F_{e}$ is the applied load vector. The super-index $\mathrm{p}$ denotes the fluid matrices, and the unknown variables are the displacement, $u_{e}$, and pressure, $P_{e}$.

\subsection{Model set-up}

\subsubsection{Model of the solid domain}

The NACA0009 hydrofoil profile was modeled within the FEM environment with a solid of the same dimensions than the actual one as shown in Fig. 5. It is important to note that PZT patches were not taken into account when modeling the mechanical properties of aluminum due to its small thickness dimension $(0.5 \mathrm{~mm})$.

A sensibility analysis of the solid domain mesh under vacuum conditions was performed to determine its optimal size. First, the number of elements in the chord direction was fixed by setting the number of partitions of the lines that enclose the lateral surface. Next, the number of partitions of the lines in the span-wise direction was varied to analyze its influence on the obtained natural frequencies and vice versa.

Fig. 6 shows the deviation in percentage of the simulated natural frequencies for various numbers of partitions in the chord and span-wise directions relative to the results obtained with 300 partitions.

As shown by the trends, the solution with no fluid domain yet defined converges quickly as the number of elements in both directions is increased. The results indicate that only a coarse mesh is required to obtain a satisfactory solution.

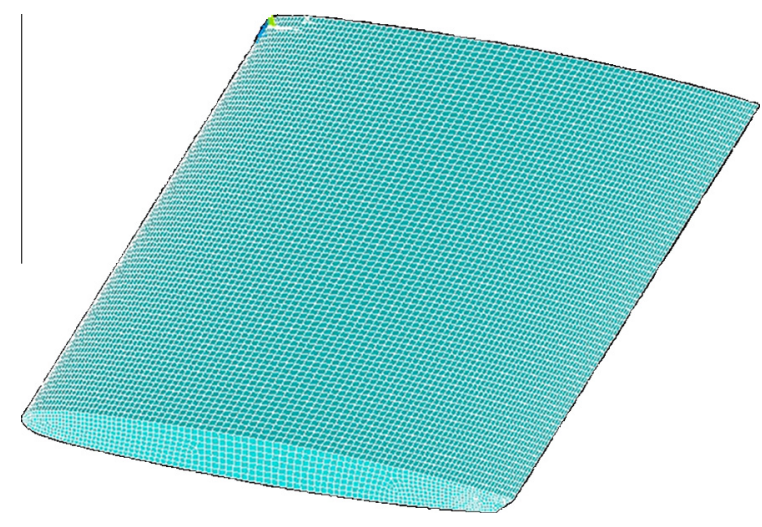

Fig. 5. FEM model of the solid NACA0009 profile.

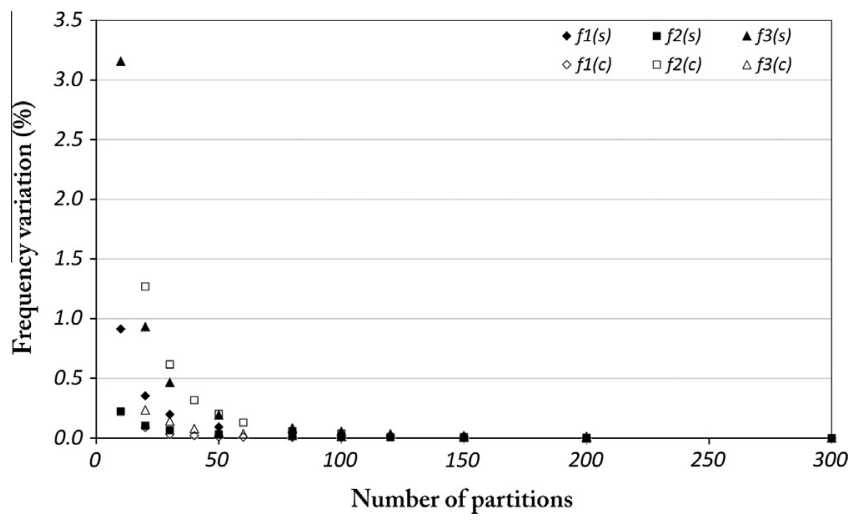

Fig. 6. Natural frequencies variation relative to results with 300 partitions (\%) against the number of partitions in the chord (c) and span-wise (s) directions for the three modes of vibration.

Table 1

Material properties set in the FEM fluid domain for air and water.

\begin{tabular}{lll}
\hline Material & Sonic velocity $(\mathrm{m} / \mathrm{s})$ & Density $\left(\mathrm{kg} / \mathrm{m}^{3}\right)$ \\
\hline Air & 343 & 1.2 \\
Water & 1482 & 998.0 \\
\hline
\end{tabular}

\subsubsection{Model of the surrounding fluid domain}

The fluid domain modeled around the solid is shown in Fig. 7. This fluid domain can be changed from Air to Water by setting the elemental material properties depending on the conditions simulated as listed in Table 1. It has to be mentioned that the orientation of the hydrofoil within the tunnel test section for the gap study was horizontal (Fig. 7) meanwhile for the partial submergence study it was vertical (Fig. 8). Therefore, two different fluid domains were defined, analyzed and used. Moreover, the gap size under normal conditions is approximately of $0.12 \mathrm{~mm}$.

Taking into account the test section dimensions in the experimental test rig (left of Fig. 8), the fluid volume is limited in two of the three axes of the problem. It is obvious that the length of the fluid domain in the horizontal axis, $h$, is not bounded by the test section (right of Fig. 8).

Since the fluid boundaries have been modeled as fully reflective, which means that they are capable of reflecting the wave pressure (Eq. (1)) with no energy loss, the location of the fluid walls with respect to the solid domain is expected to influence the numerical solution. Therefore, the influence of the dimension $h$ has been analyzed to carefully determine the final fluid domain volume. For that 


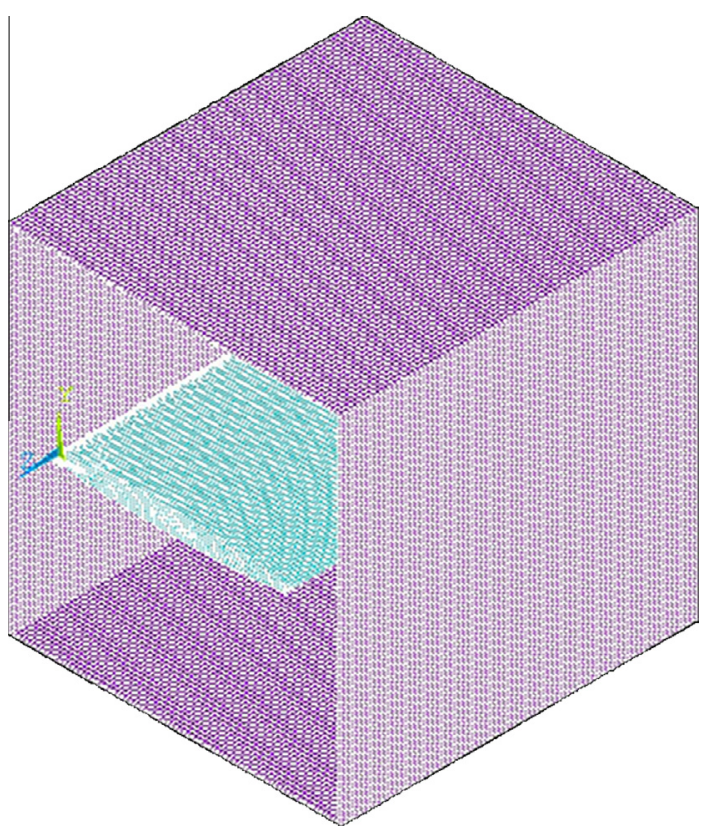

Fig. 7. NACA0009 solid domain in blue and fluid domain in purple. (For interpretation of the references to color in this figure legend, the reader is referred to the web version of this article.)

purpose, the size $h$ of the fluid volume was gradually increased in a step-by-step manner and the natural frequencies were calculated for each case with the hydrofoil vertically orientated. The observed natural frequency variation with respect to dimension $h$ has been plotted in Fig. 9. For the hydrofoil horizontal orientation, the natural frequencies behavior is much more insensible to $h$ and the variation for analogous distances falls below $0.7 \%$. Consequently, and after examining the results, a horizontal dimension $h$ of $0.13 \mathrm{~m}$ has been finally chosen to limit the fluid volume for both orientations.

Under normal conditions, the size of this gap is approximately $0.12 \mathrm{~mm}$. In this context, the gap was also modeled and the subsequent fluid domain resulted in a cubic volume of dimensions $0.15012(s) \times 0.15(l) \times 0.13(h) \mathrm{m}$ (see Fig. 8).

An additional sensibility analysis was performed within the gap dimension due to its small size relative to the hydrofoil span size. In fact, the number of fluid elements had to be substantially increased with respect to previous sensibility analysis to avoid degradation of the quality of the mesh.

Therefore, the distance from the hydrofoil tip section to the lateral wall was meshed with different numbers of fluid elements to capture the gap effect. A simplified 2D model was used for this

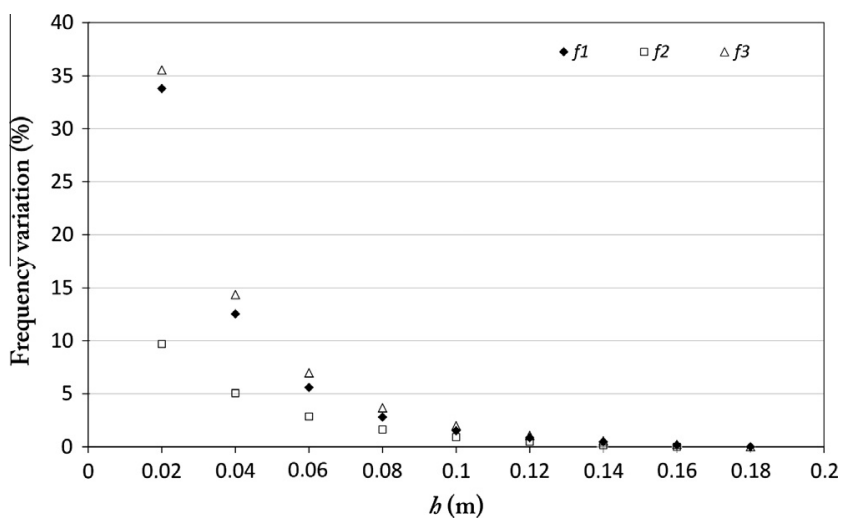

Fig. 9. Natural frequency variation in\% as a function of the horizontal dimension of the fluid domain, $h$ relative to results with $h=0.18$.

proof. As a result only the bending modes, which were expected to be those most sensitive to such effect, could be studied. The results are presented in Fig. 10, where the difference in natural frequency between the use of 1 or 10 elements just falls below $0.1 \%$. Therefore, under normal conditions (gap of $0.12 \mathrm{~mm}$ ), one unique element was eventually used to mesh the fluid located inside the gap in spanwise direction.

The selected structural element was 8-node 3D type with three degrees of freedom (displacements in all directions) which allows for prism and tetrahedral degenerations. For the current modal analysis, only the density, the Young's modulus and the Poisson coefficient were required. Moreover, the structural element was able to admit pressure loads.

The selected acoustic fluid element was 8-node 3D type with four degrees of freedom (displacements in all directions and pressure) which also which allows for prism and tetrahedral degenerations. For modal analysis, only the density and the sonic velocity were required as material properties to obtain Eq. (1). In addition, a special option was available to identify those fluid elements in contact with structural elements (interphase), producing an unsymmetrical element matrix. Surface loads (FSI) also had to be applied at the interphase.

3.2.2.1. Lateral gap analysis. The initial model dimensions were $0.15012 \times 0.15 \times 0.13 \mathrm{~m}$ and the mesh was composed of 53640 structural elements and of 610406 fluid elements. In this case, the profile was horizontally oriented.

During the experiments, the gap distance was increased from $0.12 \mathrm{~mm}$ to $2.12 \mathrm{~mm}$ by displacing the hydrofoil backwards in spanwise direction. In FEM environment, these conditions was
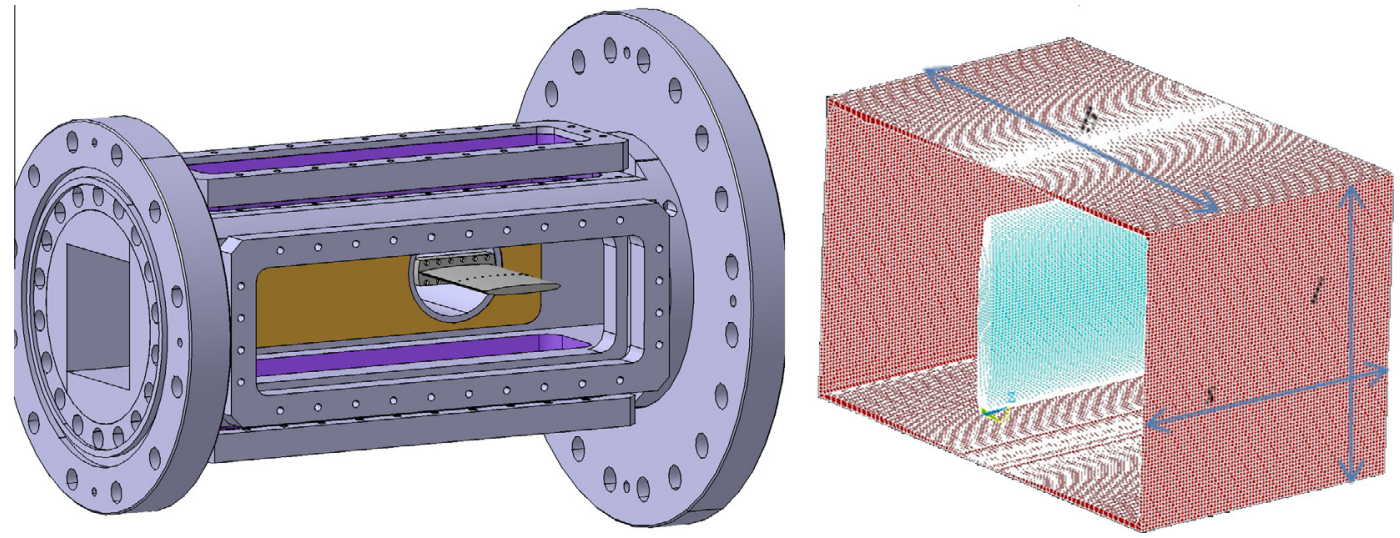

Fig. 8. Sketch of the LMH cavitation tunnel test section (left) and its model representation in FEM (right) with corresponding dimensions. 


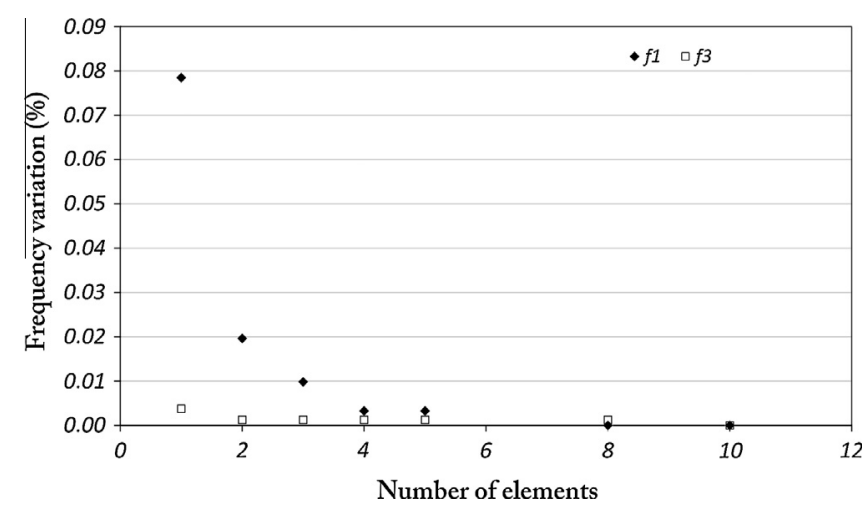

Fig. 10. Solution variation in\% plotted against the number of elements in the gap direction for $f_{1}$ (blue) and $f_{3}$ (red).

Table 2

Gap distances and number of fluid elements used to model them.

\begin{tabular}{ll}
\hline Gap distance $(\mathrm{mm})$ & Elements along gap distance \\
\hline 0.12 & 1 \\
0.22 & 1 \\
0.32 & 2 \\
0.42 & 3 \\
0.62 & 5 \\
0.72 & 6 \\
0.82 & 6 \\
1.12 & 9 \\
2.12 & 17 \\
\hline
\end{tabular}

simulated by increasing the fluid volume dimension $s$ from the original $0.15012 \mathrm{~m}$ to a final value of $0.15212 \mathrm{~m}$ in 8 steps as indicated in Table 2. Each increase of the gap distance was modeled by increasing the number of element layers accordingly.

3.2.2.2. Partially submerged hydrofoil. The fluid volume dimensions and the mesh were analogous to the ones used for the standard conditions with the gap of $0.12 \mathrm{~mm}$. In this case, the profile was vertically oriented.

During the experiments, the tunnel test section was filled stepby-step so that the free surface was located at different heights. The numerical procedure to simulate such conditions consisted of changing the material properties from Water to Air for the adequate number of elements to simulate the submergence level of the hydrofoil. In other words, the upper part of the fluid domain was Air and the lower part of the fluid domain was Water as shown in Fig. 11.

For this simulation and to avoid modeling the free surface as a fully reflective boundary, which would lead to an overestimation of the added mass results, the pressure of the Air nodes was fixed to atmospheric pressure. This setting avoids allowing the wave pressure to be reflected by the free surface because the nodes in this boundary do not have any additional flexibility in their pressure degree-of-freedom.

\section{Results and discussion}

\subsection{Lateral gap analysis}

The experimental and the simulated added mass coefficients for the three modes of vibration are listed in Table 3 and plotted as a function of gap distance in Figs. 12-14. The reference natural frequencies of the hydrofoil in air, used to obtain $C_{M}$, are $266.7 \mathrm{~Hz}$ for $f_{1}, 1008.7 \mathrm{~Hz}$ for $f_{2}$ and $1628.8 \mathrm{~Hz}$ for $f_{3}$.

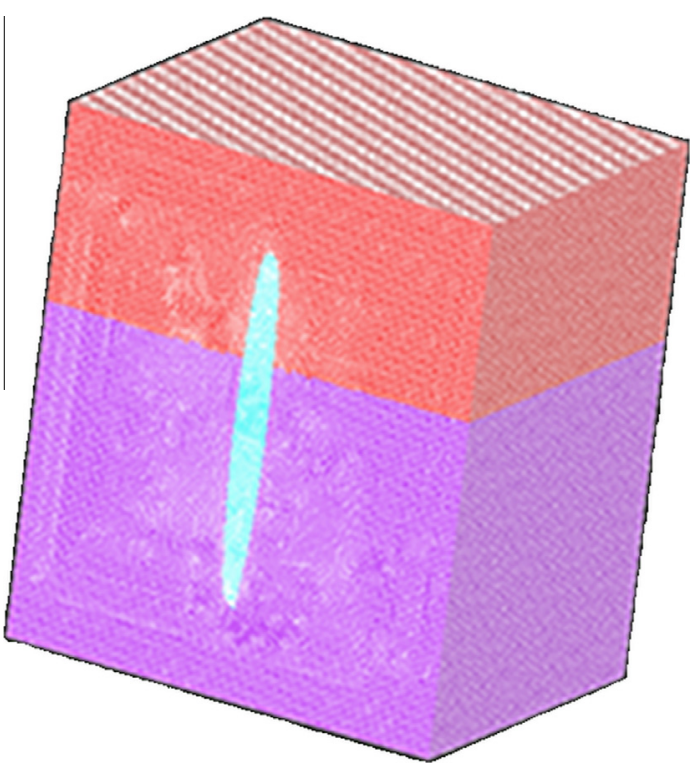

Fig. 11. Sectional view of the NACA0009 profile partially submerged in water (purple domain) and in contact with air (red domain). (For interpretation of the references to color in this figure legend, the reader is referred to the web version of this article.)

Table 3

Experimental and numerical $C_{M}$ values for different gap distances.

\begin{tabular}{llllllll}
\hline \multirow{2}{*}{ Gap (mm) } & \multicolumn{2}{l}{ Experimental } & & \multicolumn{2}{l}{ FEM } & \\
\cline { 2 - 3 } & $C_{M 1}$ & $C_{M 2}$ & $C_{M 3}$ & & $C_{M 1}$ & $C_{M 2}$ & $C_{M 3}$ \\
\hline 0.12 & 3.69 & 1.73 & 2.58 & & 3.76 & 1.63 & 2.20 \\
0.22 & 3.65 & 1.71 & 2.57 & & 3.70 & 1.62 & 2.18 \\
0.32 & 3.60 & 1.71 & 2.51 & & 3.65 & 1.61 & 2.17 \\
0.42 & 3.57 & 1.70 & 2.48 & & 3.60 & 1.61 & 2.16 \\
0.62 & 3.52 & 1.69 & 2.49 & 3.52 & 1.59 & 2.15 \\
0.72 & 3.48 & 1.67 & 2.44 & 3.49 & 1.59 & 2.14 \\
0.82 & 3.44 & 1.67 & 2.43 & & 3.46 & 1.58 & 2.13 \\
1.12 & 3.39 & 1.66 & 2.44 & 3.38 & 1.57 & 2.12 \\
2.12 & 3.24 & 1.63 & 2.38 & 3.19 & 1.54 & 2.08 \\
\hline
\end{tabular}

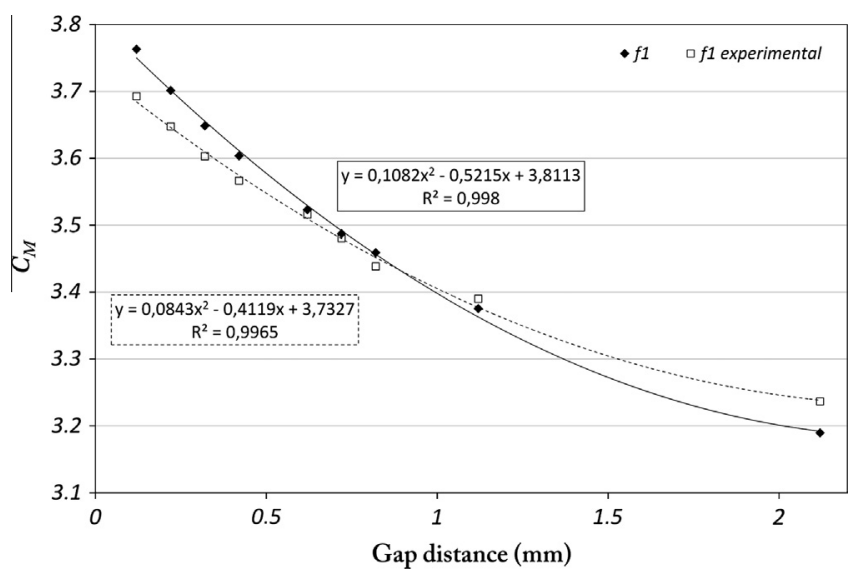

Fig. 12. Experimental and numerical $C_{M}$ values plotted against the gap size for the first bending mode.

For all the modes of vibration, the added mass coefficient increases as the gap distance is reduced. As shown, the trend of the experimental results is well captured with the simulation for all the modes of vibration. Nevertheless, for the second bending mode the coefficient $C_{M 3}$ is significantly underpredicted by a value of about 0.33 for all the cases. 


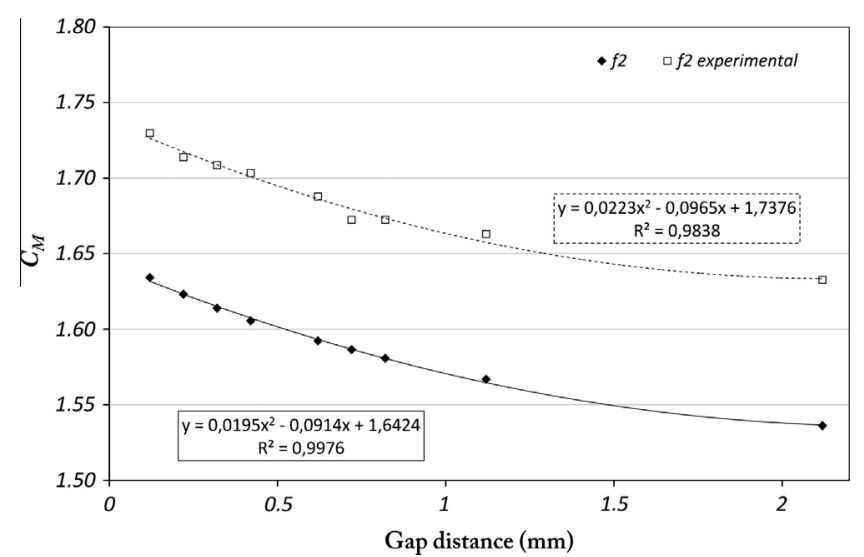

Fig. 13. Experimental and numerical $C_{M}$ values plotted against the gap size for the first torsion mode.

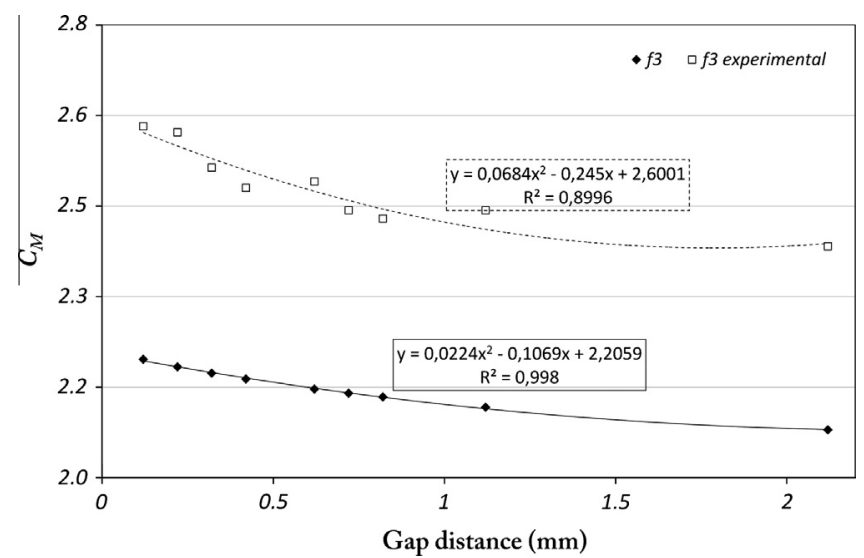

Fig. 14. Experimental and numerical $C_{M}$ values plotted against the gap size for the second bending mode.

The evolutions of the added mass coefficients are well approximated with quadratic models of the form indicated in the regression equations presented in each figure as proved by the high correlation coefficients. Based on these results, the significance of the gap effect depending on the mode shape is verified and quantified. It can be observed that both bending modes present a steeper trend than the torsion mode, which indicates that they are more affected by the presence of the solid boundary. In summary for the numerical results, the trends of the gap effects are in general well simulated even if $f_{3}$ is overestimated.

\subsection{Partially submerged hydrofoil}

The asymmetry of the hydrofoil makes it necessary to consider its orientation during the submergence tests. Therefore, the hydrofoil has been rotated to a vertical position with the trailing edge (TE) or with the leading edge (LE) at the bottom side of the test section.

The experimental and numerical added mass coefficients for the three modes of vibration obtained as a function of the percentage of hydrofoil surface (with $0 \%$ meaning that all the surface is in contact with Air and with 100\% meaning that all the is surface submerged in Water) are listed in Tables 4 and 5. These values are plotted against the submergence percentage in Figs. 15 and 16 for TE orientation and Figs. 17 and 18 for LE orientation.

The results clearly indicate that as the hydrofoil is submerged, the added mass increases for all modes. However, the evolution
Table 4

Experimental and numerical $C_{M}$ values for different submergence levels with TE orientation.

\begin{tabular}{|c|c|c|c|c|c|c|}
\hline \multirow[t]{2}{*}{ Submergence (\%) } & \multicolumn{3}{|c|}{ Experimental } & \multicolumn{3}{|l|}{ FEM } \\
\hline & $C_{M 1}$ & $C_{M 2}$ & $C_{M 3}$ & $C_{M 1}$ & $C_{M 2}$ & $C_{M 3}$ \\
\hline 100 & 2.73 & 1.57 & 2.79 & 2.73 & 1.41 & 1.86 \\
\hline 90 & 2.24 & 1.13 & 2.19 & 2.26 & 1.05 & 1.58 \\
\hline 80 & 1.75 & 0.85 & 1.55 & 1.80 & 0.80 & 1.32 \\
\hline 70 & 1.44 & 0.73 & 1.20 & 1.39 & 0.67 & 1.08 \\
\hline 60 & 1.02 & 0.60 & 0.90 & 1.03 & 0.61 & 0.86 \\
\hline 50 & 0.80 & 0.60 & 0.76 & 0.72 & 0.58 & 0.66 \\
\hline 40 & 0.52 & 0.57 & 0.54 & 0.47 & 0.54 & 0.48 \\
\hline 30 & & 0.46 & 0.30 & 0.26 & 0.44 & 0.29 \\
\hline 20 & 0.14 & 0.26 & 0.12 & 0.12 & 0.28 & 0.14 \\
\hline 10 & & 0.08 & 0.02 & 0.03 & 0.09 & 0.03 \\
\hline 0 & & 0.00 & -0.01 & 0.00 & 0.00 & 0.00 \\
\hline
\end{tabular}

Table 5

Experimental and numerical $C_{M}$ values for different submergence levels with LE orientation.

\begin{tabular}{|c|c|c|c|c|c|c|}
\hline \multirow[t]{2}{*}{ Submergence (\%) } & \multicolumn{3}{|c|}{ Experimental } & \multicolumn{3}{|l|}{ FEM } \\
\hline & $C_{M 1}$ & $C_{M 2}$ & $C_{M 3}$ & $C_{M 1}$ & $C_{M 2}$ & $C_{M 3}$ \\
\hline 100 & 2.76 & 1.55 & 2.57 & 2.73 & 1.37 & 1.86 \\
\hline 90 & 2.26 & 1.04 & 2.09 & 2.28 & 1.00 & 1.58 \\
\hline 80 & 1.77 & 0.76 & 1.52 & 1.80 & 0.72 & 1.30 \\
\hline 70 & 1.44 & 0.59 & 1.15 & 1.40 & 0.57 & 1.05 \\
\hline 60 & 1.10 & 0.53 & 0.86 & 1.03 & 0.50 & 0.83 \\
\hline 50 & 0.80 & 0.49 & 0.67 & 0.73 & 0.48 & 0.63 \\
\hline 40 & 0.51 & 0.49 & 0.46 & 0.47 & 0.44 & 0.44 \\
\hline 30 & & 0.36 & 0.24 & 0.27 & 0.37 & 0.27 \\
\hline 20 & 0.14 & 0.23 & 0.10 & 0.12 & 0.24 & 0.13 \\
\hline 10 & & 0.05 & -0.01 & 0.03 & 0.08 & 0.03 \\
\hline 0 & & 0.00 & -0.01 & 0.00 & 0.00 & 0.00 \\
\hline
\end{tabular}

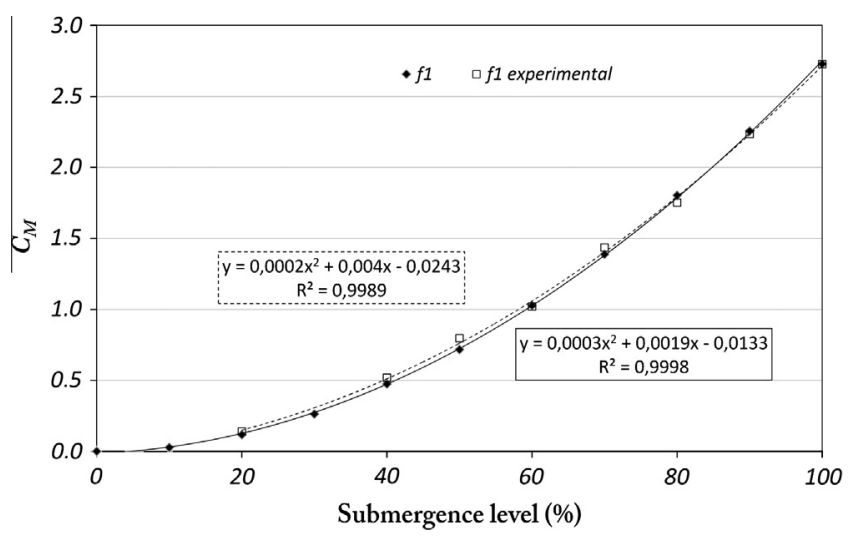

Fig. 15. Experimental and numerical $C_{M}$ values for $f_{1}$ for different submergence levels with TE orientation.

of the coefficients differs between the bending and torsion modes. For bending modes, a growing trend is observed for any submergence level whereas for the torsion mode this growing trend is reduced to a nearly constant value between $40 \%$ and $60 \%$ submergence. For the bending modes, the equations that best fit the experimental results are quadratic type as indicated in the trend equations by the high correlation coefficients. As observed from the graphs, the numerical results are close to the experimental ones, except for the second bending mode in the range from $80 \%$ to $100 \%$ submergence.

To compare the effects of the profile orientation, the torsion mode $f_{2}$ has been chosen because this particular mode shape with a nodal line in the hydrofoil center line is expected to be influenced 


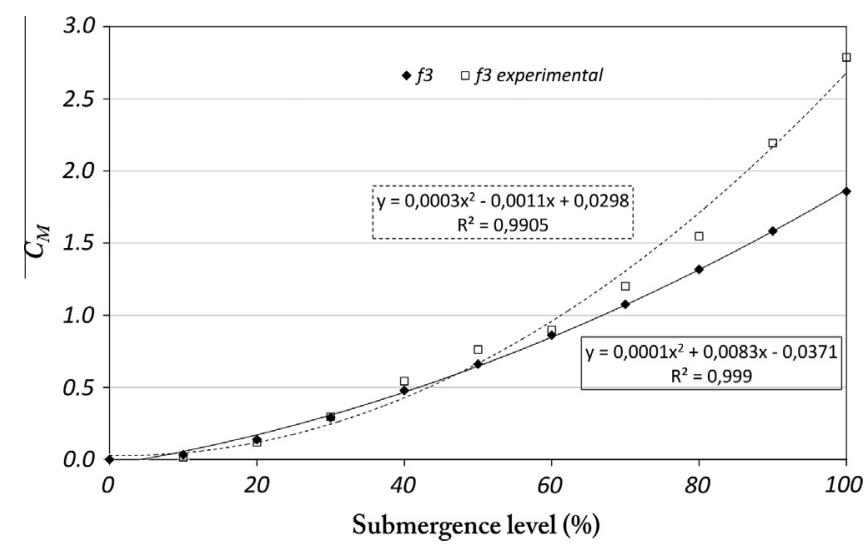

Fig. 16. Experimental and numerical $C_{M}$ values for $f_{3}$ for different submergence levels with TE orientation.

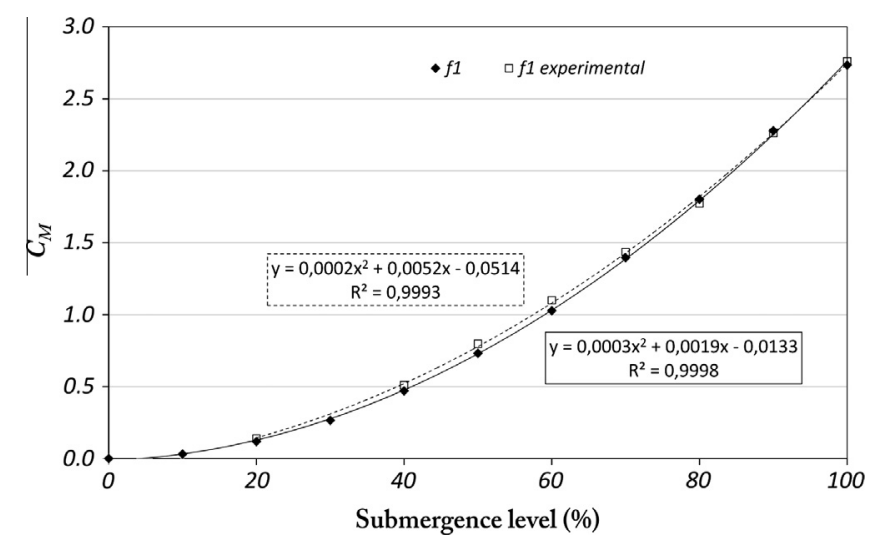

Fig. 17. Experimental and numerical $C_{M}$ values for $f_{1}$ for different submergence levels with LE orientation.

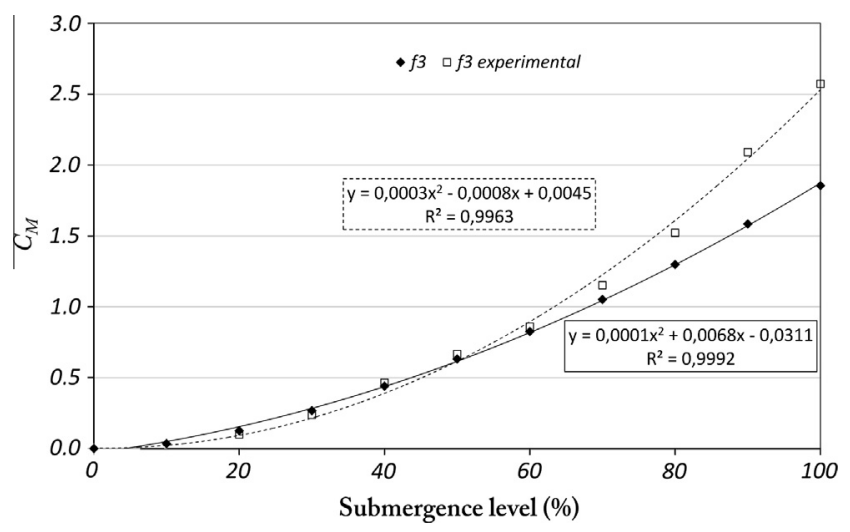

Fig. 18. Experimental and numerical $C_{M}$ values for $f_{3}$ for different submergence levels with LE orientation.

by such condition. Effectively, the $C_{M}$ values are slightly different depending on the orientation. This is observed in Fig. 19 where the numerical results for both orientations have been superposed which indicates that the trailing edge orientation experiences a greater added mass than the leading edge orientation for the same submergence levels between $20 \%$ and $80 \%$. A similar behavior is also observed in the experimental results. This phenomenon could be explained observing the geometry of both edges. The TE is significantly sharper than the LE and it suffers greater deformations

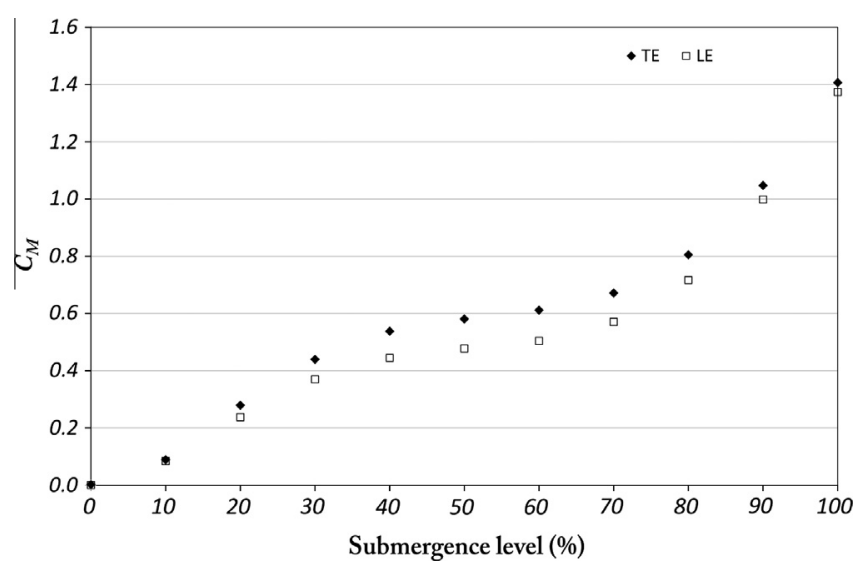

Fig. 19. $C_{M}$ values for $f_{2}$ plotted against the submergence level. Orientation comparison.

during torsion motion. These greater deformations induce higher accelerations in the surrounding fluid domain and therefore higher added mass effect.

As observed in Figs. 15-19 the variation of $C_{M}$ with the submergence level is clearly related to the fluid entrained by the mode shape motion at each condition. In particular, if we focus on the torsion mode, the shape of the evolution of the $C_{M}$ values depicts a plot of the motion itself. At the extreme submergences when most of the fluid is accelerated by the large hydrofoil displacements the results show the most significant increases in the added mass. Meanwhile, when the free surface is close to the mode nodal line at the hydrofoil centerline, the increase of added mass with submergence is lower because not much fluid is entrained due to the smaller surface displacements around this zone. Consequently, the $C_{M}$ values are nearly constant around the $50 \%$ submergence levels.

Similarly, it would be expected that for bending modes, the variation of $C_{M}$ was basically constant throughout the submergence process due to the expected parallel motion of the hydrofoil relative to the free surface. Surprisingly, the effects on the added mass are better captured with a second-order polynomial law. As observed in Figs. 15-18, for low submergence levels, the $C_{M}$ increase is quite weak whereas it is far steeper for high levels.

Regarding the submergence effects, the torsion mode results reproduce well their characteristic shape and the effect of the profile orientation is also correctly simulated (Fig. 19). From the current results, it is confirmed the well-known fact that the added mass effect on a submerged structure depends on the particular mode shape deformation that is excited, as already proved by Yadykin et al. [19].

\section{Conclusions}

The effects of two different boundary conditions on the dynamic response of a hydrofoil mounted like a cantilever beam have been numerically and experimentally investigated.

The first one has consisted in a nearby solid boundary located at different distances or gaps to the free lateral side of the hydrofoil in horizontal position.

The second one has consisted in submerging the hydrofoil at different levels in vertical position.

The FEM method has been used to simulate the three first natural frequencies of the hydrofoil in all the conditions experimentally tested in the EPFL High Speed Cavitation Tunnel. From them, the added mass coefficients have been calculated and compared. 
The obtained results show in general a good agreement between numerical and experimental methods. For the gap effect, the deviation of the natural frequencies falls below $1.5 \%$ for first bending and torsion modes and below $5 \%$ for second bending mode. Regarding the partial submergence, the deviations are limited to a maximum value of $3 \%$ for all the natural frequencies. Only the second bending mode for partial submergence levels higher than $80 \%$ shows poorer accuracy and the deviation grows up to $12 \%$. As a result, the accuracy shown by the numerical added mass coefficients under both boundary conditions would present a punctual maximum error up to $38 \%$. Nevertheless, the $C_{M}$ 's trends are also well captured with a 2nd order polynomial function as observed by correlation coefficients around 0.99 for all of the modes.

The presence of a solid boundary near a vibrating structure increases the added mass and, therefore, decreases its natural frequencies. For the present case, the increase of the added mass with the gap distance follows a quadratic law for all the modes of vibration.

The effect of the partial submergence on the hydrofoil added mass depends on the mode shape under study. As the percentage of wetted surface is increased, the added mass also increases. For both bending modes the behavior follows a quadratic law. Meanwhile, the torsion mode shows a distinct pattern. In fact, the rate of increase of the added mass with submergence level correlates with the characteristic deflection of the hydrofoil submerged part with steeper growths for larger surface displacements.

\section{References}

[1] Blevins RD. Formulas for natural frequency and mode shape. Malabar: Krieger; 1979.

[2] Brennen CE. A review of added mass and fluid inertial forces. A review of added mass and fluid inertial forces. Naval Civil Engineering Laboratory; 1982.
[3] De La Torre O, Escaler X, Egusquiza E, Farhat M. Experimental investigation of added mass effects on a hydrofoil under cavitation conditions. J Fluids Struct; 2013.

[4] Ergin A, Ugurlu B. Linear vibration analysis of cantilever plates partially submerged in fluid. J Fluids Struct 2003;17(7):927-39.

[5] Fu Y, Price WG. Interactions between a partially or totally immersed vibrating cantilever plate and the surrounding fluid. J Sound Vibr 1987;118:495-513.

[6] Huang X, Valero C, Egusquiza, Presas AE, Guardo A. Numerical and experimental analysis of the dynamic response of large submerged trashracks. Comput Fluids 2013;71:54-64.

[7] Kramer MR, Liu Z, Young YL. Free vibration of cantilevered composite plates in air and in water. Compos Struct 2013;95:254-63.

[8] Kwak MK. Axisymmetric vibration of circular plates in contact with fluid. J Sound Vibr 1991;146(3):381-9.

[9] Lamb H. Hydrodynamics. New York: Dover publications; 1945.

[10] Liang C, Tai Y, Li P. Natural frequencies of annular plates having contact with fluid. J Sound Vibr 1999;228(5):1167-81.

[11] Liang C, Liao C, Tai Y, Lai W. The free vibration analysis of submerged cantilever plates. Ocean Eng 2000;28:1225-45.

[12] Liang QW, Rodriguez CG, Egusquiza E, Escaler X, Farhat M, Avellan F. Numerical simulation of fluid added mass effect on a Francis turbine runner. Comput Fluids 2007;36:1106-18.

[13] Lindholm US, Kana DD, Chu W-C, Abramson HN. Elastic vibration characteristics of cantilever plates in water. J Ship Res 1965;9:11-22.

[14] Milne-Thomson LM. Theoretical hydrodynamics. 5th ed. New York: Dover; 1968. p. $515-41$.

[15] Motley MR, Matthew RK, Young YL. Free surface and solid boundary effects on the free vibration of cantilevered composites plates. Compos Struct 2013;96:365-75.

[16] Rodriguez CG, Egusquiza E, Escaler X, Liang QW, Avellan F. Experimental investigation of added mass effects on a Francis turbine runner in still water. J Fluids Struct 2006;22:699-712.

[17] Rodriguez CG, Flores P, Pierart FG, Contzen LR, Egusquiza E. Capability of structural-acoustical FSI numerical model to predict natural frequencies of submerged structures with nearby rigid surfaces. Comput Fluids 2012;64:117-26.

[18] Schiller FC. Wave forces on a submerged horizontal cylinder. MS thesis. Monterey, California: Naval Postgraduate School; 1971.

[19] Yadykin Y, Tenetov V, Levin D. The added mass of a flexible plate oscillating in a fluid. J Fluids Struct 2003;17:115-23.

[20] Yamamoto T, Nath JH, Slotta LS. Yet another report on cylinder drag or wave forces on horizontal cylinders. Bulletin 47, Engineering Experiment Station, Oregon State University; 1973. 